УАK 159.9.072.43

\title{
Возможности арт-терапии в коррекции волевого контроля и самооценки пациентов с химической зависимостью
}

\author{
Ирина А. Скиртач ${ }^{\boxplus}$ (D), Виктория В. Глазова ${ }^{2}$ (iD \\ 1 Южный фелеральный университет, г. Ростов-на-Аону, Россия \\ 1.2 Аонской государственный технический университет, г. Ростов-на-Аону, Россия \\ \iskirtach@sfedu.ru
}

\begin{abstract}
Аннотация
Актуальность исследования обусловлена необходимостью поиска эфрорективной стратегии реабилитации пациентов с зависимостью. Коррекция нарушений ^ичностной сореры выходит на первый п^ан, т. к. именно сложности с контролем и регуляцией собственного поведения, неалекватная самооценка становятся преградой на пути к вызАоровлению и социально-психологической аАаптации. Широко известно, что оАной из возможных стратегий коррекции Аанных психологических характеристик является использование в реабилитации методов арт-терапии. ОАнако в холе теоретического анализа литературы по теме исследования выявлено практически полное отсутствие исслеАований эффоективности и ограничений применения методов арт-терапии при различных виАах зависимости.

Среди психологических особенностей миц, как с алкогольной, так и с наркотической зависимостью, отмечают нарушение иерархии мотивов Аеятельности, низкую самооценку, неуверенность в себе, нарушение волевой регуляции поведения, манипулятивное поведение, алекситимию. Поэтому основной мишенью арт-терапии в работе с такими пациентами булет коррекция перечисленных нарушений. В Аанной статье освещены результаты изучения Аинамики показателей волевого контроля и самооценки пациентов с алкогольной и наркотической зависимостью при вк^ючении в реабилитационную программу методов арт-терапии.

В заключение слелан вывол о том, что эфорект арт-терапии у пациентов с разными виАами химической зависимости носит универсальный характер и не зависит От виАа химической аАликции в отношении показателей «настойчивость» и «самооценка», а в отношении коррекции общего показателя волевого контроля и показателя «самообладание» более выраженный эфроект Аостигнут в группе пациентов с наркотической зависимостью. Также впервые, в рамках данного исследования, бы^а установлена оптимальная продолжительность реабилитационной программы с применением метолов арт-терапии Аля Аостижения Аостоверного эфоректа.
\end{abstract}


МЕДИЦИНСКАЯ ПСИХОЛОГИЯ

\title{
КАючевые САова
}

химическая зависимость, алкогольная зависимость, наркотическая зависимость, метолы арт-терапии, коррекция, настойчивость, самообладание, волевой контроль, самооценка, реабилитация

\section{Аля цитирования}

Скиртач, И. А. и ГАазова, В. В. (2021). Возможности арт-терапии в коррекции во^евого контроля и самооценки пациентов с химической зависимостью. СевероКавказский психологический вестник, 19(1), 21-33. https://doi.org/10.21702/ncpb.2021.2

Original article

https://doi.org/10.21702/ncpb.2021.2

UDC 159.9.072.43

\section{Scope of Art Therapy in the Correction of Volitional Control and Self-esteem of Patients With Chemical Addiction}

\author{
Irina A. Skirtach ${ }^{1}$ (iD), Victoria V. Glazova ${ }^{2}$ \\ ' Southern Federal University, Rostov-on-Don, Russia \\ 1, 2 Don State Technical University, Rostov-on-Don, Russia \\ 注kirtach@sfedu.ru
}

\begin{abstract}
The relevance of this study is related to search for an effective strategy of rehabilitation patients with addiction. Correction of personality disorders is important. Violation of the regulation of behavior, inadequate self-esteem become an obstacle to recovery and socio-psychological adaptation. Art therapy methods are used to correct these psychological characteristics. But an analysis of literature on the topic of the study did not find any works devoted to evaluating effectiveness of art therapy methods for various types of addiction and limitations of their use.

For additive psychological features are characteristic: violation of hierarchy of motives of activity, low self-esteem, self-doubt, violation of volitional regulation of behavior, manipulative behavior, alexithymia. It is important to correct these features during the rehabilitation process.

This article presents the results of studying dynamics of indicators of volitional control and self-esteem of patients with alcohol and drug addiction when art therapy methods are included in the rehabilitation program.

It was found that art therapy is universal in correcting the indicators of "perseverance" and "self-esteem" in patients with different types of chemical addiction. Correction
\end{abstract}


of the general indicator of volitional control and the indicator "self-control" achieves a more pronounced effect in the group of patients with drug addiction. For the first time, within the framework of this study, the optimal duration of the rehabilitation program with the use of art therapy methods was established to achieve a reliable effect.

\section{Keywords}

chemical addiction, alcohol addiction, drug addiction, art therapy methods, correction, perseverance, self-control, volitional control, self-esteem, rehabilitation

\section{For citation}

Skirtach, I. A., \& Glazova, V. V. (2021). Scope of art therapy in the correction of volitional control and self-esteem of patients with chemical addiction. Severo-Kavkazskii psikhologicheskii vestnik (North-Caucasian Psychological Bulletin), 19(1), 21-33. https:// doi.org/10.21702/ncpb.2021.2 (in Russ.).

\section{Введение}

В настоящее время проблема зависимостей, в частности, химических, носит острый социальный характер. По последним данным официальной статистики, в России в лечебно-профилактических организациях состоят на учете 1208,6 тыс. больных с алкогольной зависимостью, что составляет почти 1 \% населения страны, и 223,1 тыс. больных наркоманией (Российский статистический ежегодник..., 2019). Не состоящих же на учете с химической зависимостью в несколько раз больше. Так, согласно данным, оглашенным во Всемирном докладе о наркотиках УНП ООН от 22 июня 2017 г., Россия занимает пятое место в мире по употреблению алкоголя и наркотиков (Доклад Международного комитета..., 2018). Масштабы проблемы употребления психоактивных веществ становятся причиной многочисленных медицинских, социологических и психологических исследований, посвященных проблемам профилактики и терапии зависимостей.

Стоит отметить, что большое внимание на сегодняшний день уделяется разработке и применению методов медикаментозной терапии абстинентного синдрома, препсихотических и психотических расстройств, выраженного влечения к психоактивным веществам, также уделяется внимание симптоматическим противорецидивным мероприятиям. Однако выходит за рамки первоочередного рассмотрения проблема личности пациента, тех особенностей, которые привели его к аддикции. Процедуры психологической реабилитации требуют уточнения рамок их возможностей и специфики в отношении различных видов химической зависимости. Запрос на психологическое сопровождение, реабилитацию и профилактику рецидивов продиктован 
неутешительной статистикой роста заболеваемости аддикциями различных видов, появлением новых психоактивных агентов.

Следует отметить, что в практике реабилитации лиц, страдающих зависимостями, широко используется арт-терапия.

Арт-терапия - направление в психотерапии, психокоррекции и реабилитации, включающее в себя методы воздействия и построения психотерапевтических отношений в контексте творческой активности. Так как частой проблемой пациентов с химической зависимостью является алекситимия, которая проявляется в снижении способности к вербализации своих эмоциональных состояний, то арт-терапия может быть оптимальным способом для человека исследовать аспекты своей жизни, которые в разговорной форме для него являются недоступными.

Многими исследователями выявлено, что арт-терапия для людей с химической зависимостью имеет преимущества перед другими видами психотерапии (Klingemann \& Klingemann, 2016; Shaffer et al., 2018; Aletraris, Paino, Edmond, Roman, \& Bride, 2014; Sharp, 2018; Tam, Shik, \& Lam, 2016; Halužan, 2012; Krebs, n.d.). Так, согласно данным Julliard (1995) и Moore (1983), в процессе арт-терапии возможен обход защитных механизмов, а в работах Holt \& Kaiser (2009) выявлено, что арт-терапия содействует облегчению выражения у зависимых эмоций, полезна для установления связи между мыслями, чувствами и поведением, обусловленным злоупотреблением психоактивными веществами, а процесс создания художественных работ способствует активизации мыслительных процессов и нормализации функций памяти. В исследованиях Feen-Calligan (2007) отмечено, что арт-терапия способствует духовному выздоровлению зависимых. Данные Aletraris et al. (2014) показали, что художественная и музыкальная терапия способствуют лечению зависимостей, но имеют различный эффект для конкретных групп пациентов; наибольшее значение обнаружено в отношении уменьшения отрицания зависимости, уменьшения сопротивления и повышения мотивации к лечению алкоголизма, стимулирования общения и улучшения его качества, побуждения пациентов к изменениям. В работе Копытина и Богачева (2008) доказано, что методы арт-терапии обладают не только определенным потенциалом, но и отдельными преимуществами в лечении и реабилитации людей, страдающих химическими зависимостями, т. к. возможность невербального раскрытия чувств обеспечивает раскрытие внутреннего мира пациентов, более высокий уровень их психологической безопасности и комфорта во время занятий, чем при использовании вербальной психотерапии (Саломатова, 2019). Эффективность изотерапии, песочной терапии и сказкотерапии доказана Зенцовой (2015) в работе с зависимыми пациентами, проходящими реабилитацию. Применение методов арт-терапии представлено в исследовании Москаленко (2019) на пациентах с зависимым 
пищевым поведением, также Нигматулиной и Мирзамухамедовой (2019) на школьниках с компьютерной зависимостью.

Несмотря на широкое применение методов арт-терапии в реабилитации наркозависимых, научных исследований ее эффективности и влияния на психологические особенности лиц, страдающих различными видами зависимостей, крайне мало.

Это обусловило цель исследования: изучение особенностей динамики показателей волевого контроля и самооценки у пациентов с разными видами зависимости при включении в реабилитацию методов арт-терапии.

Объектом исследования выступили пациенты реабилитационного центра «Решение» с алкогольной и наркотической зависимостью. Было обследовано 98 пациентов. Экспериментальная и контрольная группы были рандомизированы по возрастному, половому и нозологическому составу.

Исследование проводилось в период с мая 2019 г. по май 2020 г.

В группу наркозависимых пациентов были включены пациенты со второй стадией зависимости от героина (29 человек, из них 19 мужчин и 10 женщин) и со второй стадией зависимости от опиоидов (19 человек, из них 15 мужчин и 4 женщины). Средний возраст 30,6 \pm 6,3 года.

В группу пациентов с алкогольной зависимостью включены больные со второй стадией алкогольной зависимости (из них 39 мужчин и 11 женщин). Средний возраст 32,3 \pm 5,1 года.

Предметом исследования стали показатели волевого самоконтроля и самооценки пациентов с алкогольной и наркотической зависимостью, а также их динамика.

Исследование включало три основных этапа:

1 этап - первичное психодиагностическое исследование. На данном этапе проводились диагностика и анализ показателей волевого самоконтроля и самооценки пациентов.

Были сформированы две экспериментальные группы (группа наркозависимых - 24 человека и группа зависимых от алкоголя - 25 человек) и две контрольные группы (группа наркозависимых - 24 человека и группа зависимых от алкоголя - 25 человек).

Пациенты, вошедшие в экспериментальные группы, были включены в программу реабилитации с использованием методов арт-терапии. Пациенты, вошедшие в контрольные группы, проходили обычный курс реабилитации без использования методов арт-терапии.

2 этап - разработка и апробация программы реабилитации с использованием методов арт-терапии. Методологической основой программы явились: модель групповой интерактивной арт-терапии зависимостей, разработанная А. И. Копытиным и О. В. Богачевым, методика арт-терапии зависимостей, 
предложенная E. S. Holt и D. H. Kaiser, подход к реабилитации лиц, зависимых от ПАВ, с использованием методов арт-терапии Н. И. Зенцовой, рекомендации к проведению групповой арт-терапии Киселевой (2007).

3 этап - повторное психодиагностическое исследование и обработка данных, сравнение результатов исследуемых психологических особенностей и их динамики у пациентов с алкогольной и наркотической зависимостью в экспериментальных и контрольных подгруппах выборки, на основании чего делалось заключение об эффективности методов в целом и различиях в эффекте для разных видов химической зависимости. Процедура исследования включала в себя два повторных психодиагностических измерения: через 4 недели после начала реабилитационной программы и через 8 недель. Сравнение проводилось с результатами, полученными при первичном исследовании испытуемых.

\section{Методы}

В исследовании были использованы следующие методики: опросник волевого самоконтроля (ВСК) (Зверков и Эйдман, 1990), методика исследования самооценки личности (Будасси, 1972).

Статистические методы обработки результатов исследования: дисперсионный анализ ANOVA, критерий углового преобразования Фишера.

\section{Результаты}

В результате анализа данных трех тестовых срезов по опроснику волевого самоконтроля с помощью дисперсионного анализа ANOVA с повторными измерениями были выявлены достоверные различия $(p \leq 0,05)$ первичных и итоговых результатов изучения показателя «настойчивость», как способности заканчивать начатое дело и достигать поставленных целей, показателя «самообладание», как способности управлять своим эмоциональным состоянием, и общего показателя волевого контроля (BCK). Это подтверждают и данные попарного сравнения с помощью Post Hoc Tests (Unequal N HSD) результатов исследуемых групп испытуемых на предмет значимых различий по указанным показателям на начальном этапе исследования, через 4 недели после начала реализации программы реабилитации с использованием методов арт-терапии для экспериментальных групп и через 8 недель. Через 8 недель получены достоверно значимые различия результатов экспериментальных групп пациентов с алкогольной и наркотической зависимостью и обеих контрольных групп ( $p \leq 0,05)$. При этом отсутствие достоверных различий в результатах исследования показателя «настойчивость» между двумя экспериментальными группами позволяет сделать вывод, что эффект от реабилитационной программы носит универсальный характер, не зависящий от вида химической 
зависимости. В то время как наличие достоверных различий $(\mathrm{p} \leq 0,05)$ по показателю «самообладание» и общему показателю волевого контроля (ВСК) между экспериментальными группами при итоговом тестировании свидетельствует о том, что воздействие арт-терапии на пациентов с наркотической зависимостью носит более выраженный эффект в сравнении с пациентами, страдающими алкогольной зависимостью. Кроме того, оценка размера эффекта позволяет нам говорить о том, что реабилитационная программа оказала положительное воздействие на динамику показателей волевой регуляции, при этом выраженный эффект достигается только к 8 неделе реабилитации $(p \leq 0,05)$, в то время как на 4 неделе достоверные различия отсутствуют. На основании этого можно прийти к выводу, что лишь долгосрочная арт-терапия может быть эффективным инструментом коррекции данных показателей.

Различия показателей самооценки до и после реабилитационной программы с использованием арт-терапии оценивались с помощью критерия углового преобразования Фишера. Анализ динамики показателя «самооценка» показал, что в экспериментальной группе пациентов с зависимостью от наркотиков количество пациентов с уровнями самооценки - завышенным по невротическому типу, адекватно завышенным и адекватным - через 4 недели после начала арт-терапии не изменилось ( $p>0,05)$. Количество же пациентов с заниженным уровнем самооценки по невротическому типу значимо снизилось $(p \leq 0,05)$, а доля пациентов с заниженной адекватной самооценкой значимо увеличилась ( $p \leq 0,01)$. В контрольной группе также наблюдается уменьшение числа пациентов с заниженной самооценкой по невротическому типу и увеличение количества пациентов с адекватно заниженной самооценкой, но, в отличие от экспериментальной группы, динамика этих показателей в контрольной группе статистически незначимая ( $p>0,05)$. Через 8 недель после начала арт-терапии в экспериментальной группе наркозависимых пациентов значимо уменьшилось количество пациентов не только с низким уровнем самооценки по невротическому типу ( $p \leq 0,01)$, но и количество пациентов с завышенной самооценкой по невротическому типу $(p \leq 0,01)$. При этом если до занятий арт-терапией доля пациентов с невротически завышенной самооценкой составляла $16,7 \%$, то через 8 недель такие пациенты в экспериментальной группе обнаружены не были. Также в группе пациентов с наркотической зависимостью, включенных в арт-терапию, после 8 недель занятий значимо увеличилась доля пациентов с адекватным уровнем самооценки $(p \leq 0,01)$ и адекватно заниженной самооценкой ( $p \leq 0,01)$. Несмотря на положительную динамику, необходимо отметить, что через 8 недель после начала программы реабилитации с использованием методов арт-терапии достаточно большое количество пациентов имели, хоть и адекватную, но заниженную самооценку (33,3 \%), что требует продолжения арт-терапии в реабилитации 
пациентов с наркотической зависимостью. В контрольной группе зависимых от наркотиков через 8 недель эксперимента уменьшилось количество пациентов с адекватно завышенной и невротически заниженной самооценкой, увеличилось количество пациентов с адекватным и адекватно заниженным уровнем самооценки. Но и как через 4 недели, динамика самооценки в этой группе незначимая ( $p>0,05)$.

В экспериментальной группе зависимых от алкоголя через 4 недели после начала реабилитационной программы наблюдается уменьшение количества пациентов с невротически завышенной и невротически заниженной, а также с адекватно заниженной самооценкой, и увеличение количества пациентов с адекватной самооценкой, но динамика этих показателей в экспериментальной группе больных алкоголизмом незначимая ( $p>0,05)$. В контрольной группе через 4 недели реабилитации не изменилось количество пациентов с завышенной и заниженной самооценкой по невротическому типу и с адекватно завышенной самооценкой, но увеличилось число пациентов с адекватно заниженной и уменьшилось с адекватной самооценкой ( $p>0,05)$. Через 8 недель (по факту завершения реабилитационной программы) в экспериментальной группе пациентов, страдающих алкоголизмом, значительно уменьшилось количество пациентов с самооценкой, завышенной по невротическому типу $(p \leq 0,01)$ и количество пациентов с невротически заниженной самооценкой $(p \leq 0,01)$. Также в экспериментальной группе больных алкоголизмом через 8 недель после начала реабилитационной программы значимо увеличилось количество пациентов с адекватным уровнем самооценки $(p \leq 0,01)$. Если до проведения арт-терапии доля пациентов, страдающих алкоголизмом, с адекватной самооценкой составляла лишь $8 \%$, то через 8 недель занятий она увеличилась до 52 \%. В контрольной группе по-прежнему не изменилось количество пациентов с невротически заниженной самооценкой, а динамика других уровней самооценки и через 8 недель реабилитации без применения арт-терапии незначительная ( $p>0,05)$.

\section{Обсуждение результатов}

Результаты анализа динамики показателей волевого контроля и самооценки показали, что программа реабилитации с использованием методов арт-терапии способствует повышению настойчивости, самообладания и общего показателя волевого контроля, формированию адекватной самооценки у пациентов с химическими зависимостями. Полученные результаты согласуются с данными исследований других авторов. Так, Зенцовой (2015) после применения модели арт-терапии в реабилитации пациентов с наркотической и алкогольной зависимостью получены более низкие показатели подозрительности и более высокие показатели саморегуляции эмоций и волевого контроля 
в экспериментальных группах. По наблюдениям Holt \& Kaiser (2009), в процессе арт-терапии у пациентов с химическими зависимостями повышаются самооценка и уверенность в себе. Копытин и Богачев (2008) утверждают, что изотерапия повышает у пациентов с химическими зависимостями самооценку.

Данных о влиянии арт-терапии на другие исследуемые нами психологические особенности пациентов с химическими зависимостями в доступной литературе не обнаружено. Также отсутствуют указания на эффекты краткосрочной и долгосрочной арт-терапии и различия в динамике психологических особенностей у пациентов с разными видами химических зависимостей в процессе арт-терапии.

Проведенное исследование восполняет этот пробел и дополняет существующие представления о проблеме реабилитации пациентов с химическими зависимостями. Так, доказано, что как у пациентов с алкогольной зависимостью, так и у наркозависимых пациентов для получения статистически достоверного эффекта от реабилитационной программы с использованием методов арт-терапии в коррекции всех исследуемых показателей волевой регуляции необходима ее продолжительность не менее 8 недель. Это объясняется как трудностью формирования положительных личностных качеств у взрослых людей вследствие возрастных особенностей и повышения ригидности под влиянием аддикции, так и наличием нарушений, появившихся вследствие злоупотребления психоактивными веществами. Так, нарушения могут затрагивать области префронтальной коры, ответственные за планирование, контроль эмоций, участвующие в волевой регуляции. Как перестройка рецепторного мозгового аппарата, которая становится мишенью при химических аддикциях, формирование новых паттернов поведения, так и выстраивание адекватного отношения и доверия к себе требуют времени и тщательной проработки (Лопатин и Лопатина, 2017). Поэтому, когда речь идет о химических аддикциях, не стоит ждать быстрых результатов. В целом же следует отметить, что арт-терапия оказывает более выраженный реабилитационный эффект на группу пациентов с наркотической зависимостью.

Полученные результаты показывают, что программа реабилитации с использованием методов арт-терапии является эффективной в работе с пациентами с разными видами химической аддикции, но при ее проведении необходимо учитывать, что положительный эффект у этой категории пациентов появляется не менее чем через 8 недель.

\section{Заключение}

Таким образом, в ходе исследования выявлено, что арт-терапия может способствовать коррекции психологических особенностей у зависимых пациентов: у пациентов с химическими зависимостями, независимо от их вида, 
МЕДИЦИНСКАЯ ПСИХОЛОГИЯ

получена значимая положительная динамика волевой регуляции поведения и самооценки.

Доказано, что динамика психологических особенностей может отличаться у людей с разными видами зависимости при включении в реабилитацию методов арт-терапии: в коррекции волевого контроля программа реабилитации с использованием методов арт-терапии при наркотической зависимости дает более выраженный эффект, чем у пациентов с алкогольной зависимостью. В отношении остальных показателей эффект носит универсальный характер.

Выявлено, что эффект арт-терапии в коррекции психологических особенностей у зависимых пациентов может зависеть от продолжительности ее проведения: краткосрочный эффект арт-терапии у пациентов с химической зависимостью проявляется в повышении способности к волевому самоконтролю эмоций и коррекции низкой самооценки, тогда как изменение способности к контролю поведения и деятельности, волевого контроля и коррекция неадекватно завышенной самооценки являются эффектом более длительной арт-терапии.

\section{Литература}

Будасси, С. А. (1972). Методика исследования самооченки личности. Москва: Академия.

Доклад Международного комитета по контролю над наркотиками за 2017 год (2018). Вена: ООН.

Зверков, А. Г. и Эйдман, Е. В. (1990). Диагностика волевого самоконтроля (опросник ВСК). В Практикум по психодиагностике. Психодиагностика мотивации и саморегуляции (с. 116-126). Москва: МГУ.

Зенцова, Н. И. (2015). Системная модель психологического этапа реабилитации больных наркоманией (докторская диссертация). Московский государственный университет им. М. В. Ломоносова, Москва.

Киселева, М. В. (2007). Арт-терапия в практической психологии и сочиальной работе. Санкт-Петербург: Речь.

Копытин, А. И. и Богачев, О. В. (2008). Арm-терапия наркоманий. Лечение, реабилитация, постреабилитация. Москва: Психотерапия.

Лопатин, В. и Лопатина, Т. (2017). Применение глицина при лечении алкоголизма. Врач, 7, 41-42.

Москаленко, А. Е. (2019). Арт-терапия в работе с зависимым пищевым поведением. В О. А. Холина (ред.), Актуальные проблемы профилактики аддиктивного поведения (с. 261-264). Ростов-на-Дону: Ростовский государственный экономический университет «РИНХ».

Нигматулина, Л. А. и Мирзамухамедова, К. (2019). Методы арт-терапии при коррекции компьютерной зависимости у обучающихся в школе. В І. С. Попович, 
С. І. Бабатіна, І. Р. Крупник (ред.), Інсайт: психологічні виміри суспільства: матер. міжнар. конф. (Вип. 1, с. 209-211). Херсон: ВД «Гельветика».

Российский статистический ежегодник. 2019: Cmam. сб. / Росстат (2019). Москва.

Саломатова, Е. А. (2019). К вопросу о понятии и специфике профилактики нехимических аддикций. Вестник науки и образования, 15, 104-107.

Aletraris L., Paino, M., Edmond, M. B., Roman, P. M., \& Bride, B. E. (2014). The use of art and music therapy in substance abuse treatment programs. Journal of Addictions Nursing, 25(4), 190-196. https://doi.org/10.1097/JAN.0000000000000048

Feen-Calligan, H. (2007). The use of art therapy in detoxification from chemical addiction. Canadian Art Therapy Association Journal, 20(1), 16-28. https://doi. org/10.1080/08322473.2007.11432289

Halužan, M. (2012). Art therapy in the treatment of alcoholics. Alcoholism and Psychiatry Research: Journal on Psychiatric Research and Addictions, 48(2), 99-105.

Holt, E. S., \& Kaiser, D. H. (2009). The First Step Series: Art therapy for early substance abuse treatment. The Arts in Psychotherapy, 36(4), 245-250. https:// doi.org/10.1016/j.aip.2009.05.004

Julliard, K. (1995). Increasing chemically dependent patients' belief in Step One through expressive therapy. American Journal of Art Therapy, 33(4), 110-119.

Klingemann, J., \& Klingemann, H. (2016). Beyond narratives: "Free drawings" as visual data in addiction research. Substance Use \& Misuse, 51(6), 682-691. https://doi.org/10.3109/10826084.2015.1135951

Krebs, K. A. (n.d.). Art therapy used to enhance steps one, two and three of a twelvestep recovery program for addictions treatment. Retrieved from https://www. researchgate.net/publication/265040798 Art Therapy Used to Enhance Steps_One_Two_and_Three_of_a_Twelve-step_Recovery_Program_for Addictions Treatment

Moore, R. (1983). Art therapy with substance abusers: A review of the literature. The Arts in Psychotherapy, 10, 251-260.

Shaffer, H. J., Tom, M. A., Wiley, R. C., Wong, M. F. Y., Chan, E. M. L., Cheng, G. L. F., .. Lee, M. (2018). Using the Syndrome Model of Addiction: A preliminary consideration of psychological states and traits. International Journal of Mental Health and Addiction, 16(6), 1373-1393. https://doi.org/10.1007/s11469-018-9952-2

Sharp, M. (2018). Art therapy and the recovery process: A literature review. Expressive Therapies Capstone Theses. 30. Retrieved from https://digitalcommons.lesley. edu/expressive theses/30

Tam, H., Shik, A. W., \& Lam, S. S. (2016). Using expressive arts in relapse prevention of young psychotropic substance abusers in Hong Kong. Children and Youth Services Review, 60, 88-100. https://doi.org/10.1016/j.childyouth.2015.11.022 
МЕДИЦИНСКАЯ ПСИХОЛОГИЯ

\section{References}

Aletraris L., Paino, M., Edmond, M. B., Roman, P. M., \& Bride, B. E. (2014). The use of art and music therapy in substance abuse treatment programs. Journal of Addictions Nursing, 25(4), 190-196. https://doi.org/10.1097/JAN.0000000000000048

Budassi, S. A. (1972). Methodology of research of self-assessment of the personality. Moscow: Akademiya. (in Russ.).

Feen-Calligan, H. (2007). The use of art therapy in detoxification from chemical addiction. Canadian Art Therapy Association Journal, 20(1), 16-28. https://doi. org/10.1080/08322473.2007.11432289

Halužan, M. (2012). Art therapy in the treatment of alcoholics. Alcoholism and Psychiatry Research: Journal on Psychiatric Research and Addictions, 48(2), 99-105.

Holt, E. S., \& Kaiser, D. H. (2009). The First Step Series: Art therapy for early substance abuse treatment. The Arts in Psychotherapy, 36(4), 245-250. https:// doi.org/10.1016/j.aip.2009.05.004

Julliard, K. (1995). Increasing chemically dependent patients' belief in Step One through expressive therapy. American Journal of Art Therapy, 33(4), 110-119.

Kiseleva, M. V. (2007). Art therapy in practical psychology and social work. Saint Petersburg: Rech'. (in Russ.).

Klingemann, J., \& Klingemann, H. (2016). Beyond narratives: "Free drawings" as visual data in addiction research. Substance Use \& Misuse, 51(6), 682-691. https://doi.org/10.3109/10826084.2015.1135951

Kopytin, A. I., \& Bogachev, O. V. (2008). Art therapy of drug addiction. Treatment, rehabilitation, post-rehabilitation. Moscow: Psikhoterapiya. (in Russ.).

Krebs, K. A. (n.d.). Art therapy used to enhance steps one, two and three of a twelve-step recovery program for addictions treatment. Retrieved from https://www.researchgate. net/publication/265040798_Art_Therapy_Used_to_Enhance_Steps_One_Two and Three of a Twelve-step Recovery Program for Addictions Treatment

Lopatin, V., \& Lopatina, T. (2017). Use of glycine in the treatment of alcoholism. Vrach (The Doctor), 7, 41-42. (in Russ.).

Moore, R. (1983). Art therapy with substance abusers: A review of the literature. The Arts in Psychotherapy, 10, 251-260.

Moskalenko, A. E. (2019). Art therapy in working with dependent food behavior. In O. A. Kholina (Ed.), Actual problems of prevention of addictive behavior (pp. 261-264). Rostov-on-Don: Rostov State University of Economics. (in Russ.).

Nigmatulina, L. A., \& Mirzamukhamedova, K. (2019). Methods of art therapy in the correction of computer dependence in students at school. In I. S. Popovich, S. I. Babatina, I. G. Krupnik (Eds.), Insight: Psychological dimensions of society: Materials of the International Conference (Issue 1, pp. 209-211). Kherson: Gel'vetika. (in Russ.). 
Report of the International narcotics control Board for 2017 (2018). Vienna: UN. (in Russ.).

Russian Statistical Yearbook. 2019: Statistical collection / Russian statistics (2019). Moscow. (in Russ.).

Salomatova, E. A. (2019). On the concept and specificity of prevention of nonchemical addictions. Vestnik nauki i obrazovaniya (Bulletin of Science and Education), 15, 104-107. (in Russ.).

Shaffer, H. J., Tom, M. A., Wiley, R. C., Wong, M. F. Y., Chan, E. M. L., Cheng, G. L. F., .. Lee, M. (2018). Using the Syndrome Model of Addiction: A preliminary consideration of psychological states and traits. International Journal of Mental Health and Addiction, 16(6), 1373-1393. https://doi.org/10.1007/s11469-018-9952-2

Sharp, M. (2018). Art therapy and the recovery process: A literature review. Expressive Therapies Capstone Theses. 30. Retrieved from https://digitalcommons.lesley. edu/expressive theses/30

Tam, H., Shik, A. W., \& Lam, S. S. (2016). Using expressive arts in relapse prevention of young psychotropic substance abusers in Hong Kong. Children and Youth Services Review, 60, 88-100. https://doi.org/10.1016/j.childyouth.2015.11.022

Zentsova, N. I. (2015). System model of the psychological stage of rehabilitation of patients with drug addiction (Doctoral disertation). Lomonosov Moscow State University, Moscow. (in Russ.).

Zverkov, A. G., \& Eidman, E. V. (1990). Diagnosis of volitional self-control (VSK questionnaire). In Workshop on psychological diagnostics. Psychodiagnostics of motivation and self-regulation (pp. 116-126). Moscow: Moscow State University. (in Russ.). 